\title{
PREDICTING HOSPITAL READMISSION FOR PATIENTS WITH COPD USING NATURAL LANGUAGE PROCESSIN
}

\author{
Aavulla Ravi ${ }^{1}$, Chillivelli Vinay Kumar Sharma ${ }^{2}$, Devireddy Anurag Reddy ${ }^{3}$, Elety \\ Naveen Kumar Reddy ${ }^{4}$ \\ ${ }^{1}$ Associate Professor, Department of computer science and engineering, Guru Nanak \\ Institutions Technical Campus, Ibrahimpatnam, Telangana, India \\ ${ }^{2}$ Department of computer science and engineering, Guru Nanak Institutions Technical \\ Campus, Ibrahimpatnam, Telangana, India \\ ${ }^{3}$ Department of computer science and engineering, Guru Nanak Institutions Technical \\ Campus, Ibrahimpatnam, Telangana, India \\ ${ }^{4}$ Department of computer science and engineering, Guru Nanak Institutions Technical \\ Campus, Ibrahimpatnam, Telangana, India.
}

\section{Abstract:}

With the entry of government enactment numerous therapeutic foundations are currently in charge of achieving target readmission rates. Constant ailments represent numerous clinic readmissions and Chronic Obstructive Pulmonary Disease has been as of late added to the rundown of illnesses for which the United States government punishes healing centers bringing about unreasonable readmissions. Despite the fact that there have been endeavours to measurably anticipate those most in threat of readmission, few have concentrated basically on unstructured clinical notes. We have proposed a structure which utilizes Natural Language Processing to break down clinical notes and foresee readmission. Numerous calculations inside the field of information mining and machine learning exist, so a structure for segment choice is made to choose the best segments. Naive Bayes utilizing Chi-Squared element choice offers a quick computational circumstance.

Keywords: chronic obstructive pulmonary disease, hospital readmission, Naive Bayes, Natural language processing

\section{INTRODUCTION}

Natural Language Processing is an ongoing research topic developed in many systems. Early research systems implemented NLP tasks without the assistance of software libraries. As the field matured, libraries and toolkits became available. These software components are aimed at being reusable so that well studied tasks are not actualized literary preparation each time a framework is created. The principle target of the task is to give the best administrations utilizing Natural Language Processing. It is utilized to investigate clinical notes and anticipate readmission. We introduce a privacy-preserving patient re-admit summary retrieval protocol in our system.

The American Recovery and Reinvestment Act (ARRA) of 2009 accentuated the selection of wellbeing data innovation through the Health Information Technology for Economic and Clinical Health Act (HITECH Act).Two prime segments identified with this demonstration are: (1) Introduction of punishments for doctor's facilities for quiet readmission inside 30,60 and 90 day time span for particular determinations; (2) Introduction of the idea of Clinical Decision Support Systems (CDSS) in Electronic Health Records through "Meaningful Use" (MU) consistence. As of now, the MU consistence requires an exceptionally essential 
DOI: https://dx.doi.org/10.26808/rs.ca.i8v2.16 International Journal of Computer Application (2250-1797)

Issue 8 Volume 2, March-April 2018

execution of lead based choice emotionally supportive networks which could be presented by an office-hone doctor in light of the mix of socioeconomics, lab comes about, meds, sensitivity, and past therapeutic history.

Chronic Obstructive Pulmonary Disease has been recently added to the list of diseases for which the United States government punishes healing centers bringing about unreasonable readmissions. In spite of the fact that there have been endeavours to factually anticipate those most in risk of readmission, few have concentrated basically on unstructured clinical notes.

Existing strategies are patient readmission, regardless of methodology. Direct comparison of methodologies in the field of health informatics can be difficult. Unlike other domains, data is often restricted and cannot be released publicly. The major drawbacks of the existing methodology is the "Curse of dimensionality." Certain mathematical techniques used in low dimensional space become less effective in high dimensions.

\section{SURVEY}

The federal legislation law penalizes excessive 30-day readmission, many systematic researches are made in 2011[1]. Recent procedures center around 30-day readmissions. screening instrument for the recognition of potential cases is important to put forth advance defense administration more proficient [2]. Improve of capacity to foresee 30-day readmission after percutaneous coronary mediation from a formerly approved registry-based model. attributes of patients got from the survey of EHR can be utilized to characterize expectation for healing center readmission. The HITECH Act stipulates that health care providers demonstrate the important utilization of wellbeing IT. As a component of this demonstration, CMS identified "doctor's facility readmissions for COPD" as an expensive issue that should be tended to in the United States in general [4]. Patient data in hospitals includes a significant amount of unstructured data. Examples include physician's notes, discharge summaries, and x-ray radiology reports [5]. Chronic Obstructive Pulmonary Disease (COPD) is also one of the leading causes of disability and mortality worldwide, Hospital readmission is an important quality of care indicator [6]. In 2011 Open NLP was created as a set of Java interfaces to develop standard API for NLP tasks [7]. The aim of this Open NLP is to provide a set of libraries for well-studied tasks of NLP [8]. frameworks play an important and useful role in processing of NLP [9]. The earliest applications of NLP is medical domain [10]. Recent researches uses Apache cTAKES to annotate unstructured EHR[11].

\section{PROPOSED SYSTEM}

We have proposed a structure which utilizes Natural Language Processing to investigate clinical notes and foresee readmission. We present a security saving best k-illness names recovery convention in our framework.

\section{Algorithms:}

a) Naïve Bayes classification, is straight forward probabilistic classifier based upon the basic Bayes Theorem with an assumption of independence among predictors. The classifier assumes independence between features. Bayes classification needs less training dataset and it is a simple probabilistic classifier. It is highly scalable. 
b) Random Forest, is an ensemble algorithm which creates multiple decision trees by randomly choosing a set of features to use for each tree. Random Forest are extremely flexible and have very high accuracy.

c) $\mathbf{K}$ nearest neighbours, is widely used in pattern recognition. K-Nearest Neighbours $(\mathrm{kNN})$ is a non-parametric calculation which utilizes a separation capacity to discover the occurrences which are most like the present. This is based on learning by analogy i.e., by comparing a given test records with training records that are similar to it.

\section{User Interface}

\section{IMPLEMENTATION}

This is the first module of our project. In this the application user's first create their account properly which are stored at the back end for verification or for providing security to the accounts. If user wants to get into his account first they have to submit their constraints such as username, password and so on, otherwise can't able to access the account.

\section{Admin}

In this project the admin will handle the hospital data. And the admin view the patient details like patient admit history, patient discharge history and patient re admit summary. And also view the doctor's details as well as add new doctors into hospital.

\section{Patient}

In this project, patient first register into site then only able to upload admit details each and every time when he/she admitting into hospital and also he/she view the admit details, discharge details and re admit summary which is instructed by the doctor who discharged him before.

\section{Doctor}

In this project the doctor will take care about patient. The doctor will dictate to patients and instruct the patients to recover soon and also discharge the patients who are taking treatment under that particular doctor. Also the doctor can see the details of patients under him like view the admit history, discharge history as well as admitted patients. Additionally, he can view the patients re admit summary who are taking the treatment under him.

\section{Natural Language Processing}

In this project the National Language Processing is used to get the patients related to COPD and get re admit dates from the instructions in discharge form and check the patient was re admitted or not

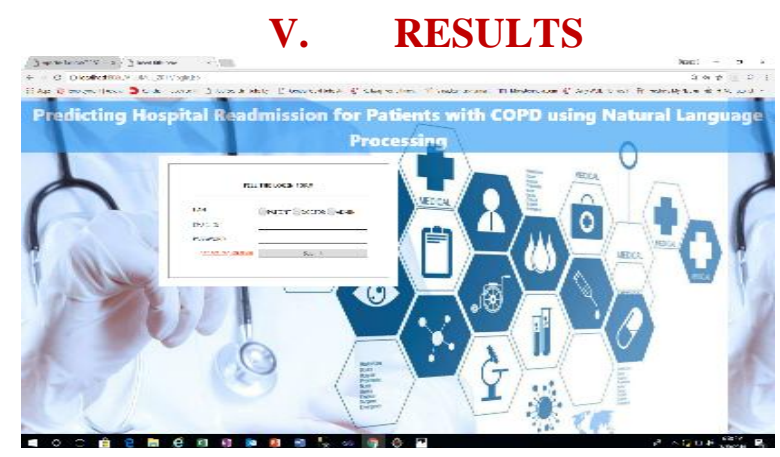

Fig.1 Design for login page 
DOI: https://dx.doi.org/10.26808/rs.ca.i8v2.16 International Journal of Computer Application (2250-1797)

Issue 8 Volume 2, March-April 2018

Figure 1 Illustrates the login page where we come across of three different modules such as the Admin, Doctor, Patient. Where each individual need to login into their respective fields with help of their mail Id and password. If the credentials are valid then the individual can login if not he/she can't.

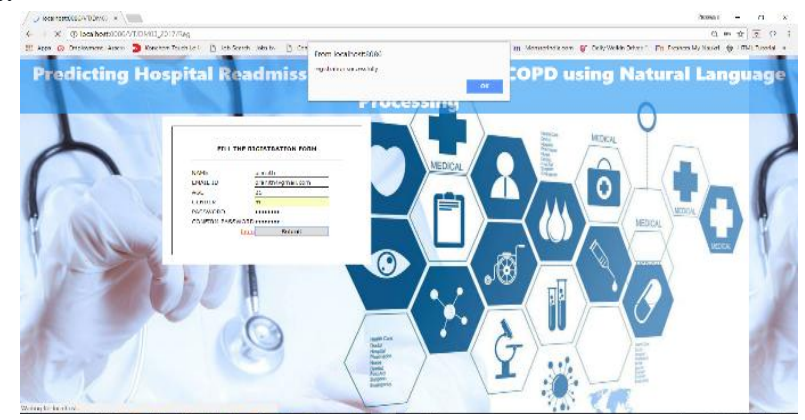

Fig.2 Design for adding patients

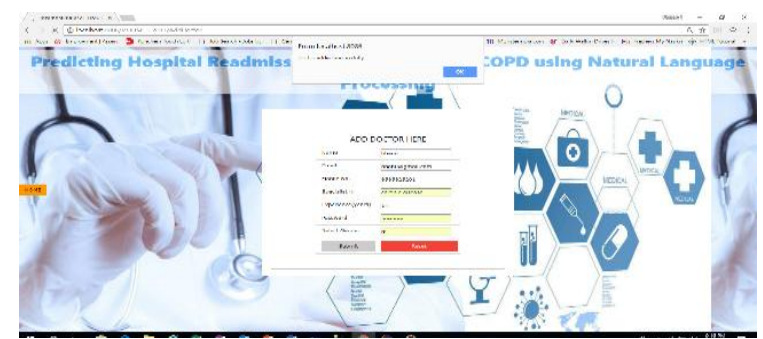

Fig.3 Design for adding doctors

Figure 2 and Figure 3 gives us the design for adding patients and doctors details into the database which is carried out by admin.

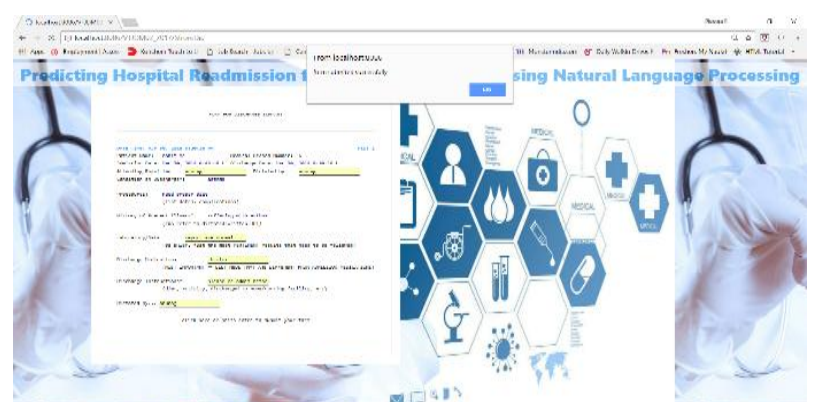

Fig.4 Design for discharge form

Figure 4 illustrates the discharge form which contains different details and discharge instructions given by the doctor when to readmit.

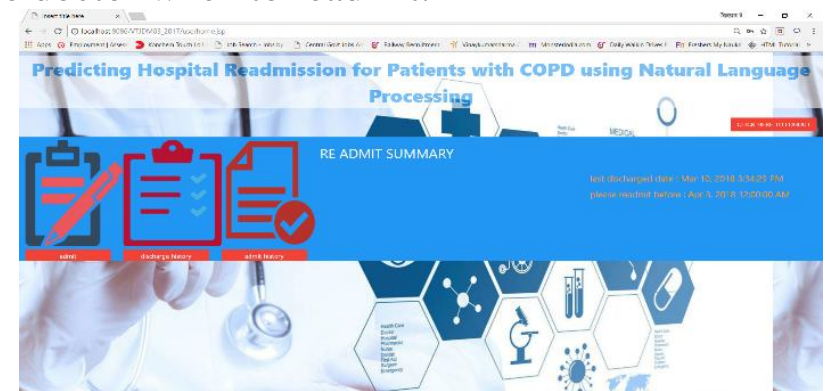

Fig.5 Design for patient home page

The above figure shows us the date when the patient need to be re-admitted. Fig 4 and Fig 5 shows the working of the NLP where the doctor mentions some sentence form information of 
DOI: https://dx.doi.org/10.26808/rs.ca.i8v2.16 International Journal of Computer Application (2250-1797)

Issue 8 Volume 2, March-April 2018

re-admission of patients, NLP transforms the data and gives us the appropriate information about the re-admit date.

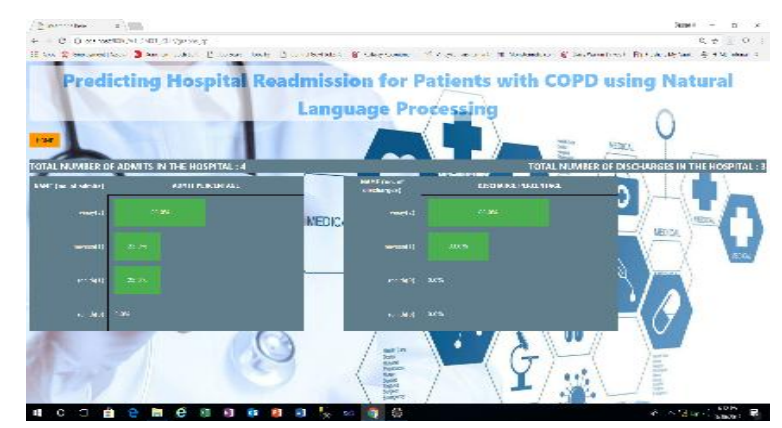

Fig.6 Design for Graphical Representation

Figure 6 Illustrates the graphical representation of the overall patients admitted and discharged and this information is used to analyse the data about rate of readmission of patients

\section{CONCLUSION}

Our readmission analysis system represents a natural language approach to patient readmission prediction. Components were evaluated and it was found that using NB classifier, selecting around $15 \%$ of the full feature set to be most effective. The system was able to predict hospital readmissions, better than existing systems. Our approach offers the advantage that separate data collection is not required for readmission prediction since clinical notes are already collected by medical institutions.

\section{FUTURE ENHANCEMENT}

With the increase in EHR systems, clinical notes will become increasingly important and NLP techniques will need to be considered when creating decision support systems. The results have shown the importance of feature selection and model creation time to the implementation of practical systems. Future work intents to extend efforts to other chronic diseases as records become available.

\section{REFERENCES}

[1] D. Kansagara et al., "Risk prediction models for hospital readmission: A systematic review," JAMA, vol. 306, no. 15, pp. 1688-1698, 2011.

[2] R. Wallmann, J. Llorca, I. G'omez-Acebo, 'A. C. Ortega, F. R. Roldan, and T. DierssenSotos, "Prediction of 30-day cardiac-related-emergency readmissions using simple administrative hospital data," Int. J. Cardiol., vol. 164, no. 2, pp. 193-200, 2013.

[3] J. H. Wasfy et al., "Enhancing the prediction of 30-Day readmission after percutaneous coronary intervention using data extracted by querying of the electronic health record," Circulation Cardiovascular Quality Outcomes, vol. 8, no. 5, pp. 477-485, 2015.

[4] D. Goodman, E. Fisher, and C. Chang, "The revolving door: A report on US hospital readmissions," Robert Wood Johnson Found., Princeton, NJ, USA, 2013.

[5] R. Behara, A. Agarwal, V. Rao and C. Baechle, "Predicting the occurrence of diabetes using analytics," in Proc.Annu.Conf.Models Appl.Decis.Sci., 2016, pp. 187-193.

[6] R. Behara, A. Agarwal, F. Fatteh, and B. Furht, "Predicting hospital readmission risk for COPD using EHR information," in Handbook of Medical and Healthcare Technologies. New York, NY, USA: Springer, 2013, pp. 297-308. 
DOI: https://dx.doi.org/10.26808/rs.ca.i8v2.16 International Journal of Computer Application (2250-1797) Issue 8 Volume 2, March-April 2018

[7] "About Grok," [Online]. Available: https://wiki.apache.org/incubator/ Open NLP Proposal. Accessed on: Jul. 6, 2016.

[8] G. S. Ingersoll, T. S. Morton, and A. L. Farris, Taming Text: How to Find, Organize, and Manipulate It.. Greenwich, CT, USA: Manning Publications Co., 2013.

[9] S. Bethard, P. V Ogren, and L. Becker, "Clear TK 2.0: Design patterns for machine learning in UIMA," in Proc. 9th Int. Conf. Lang. Resources Eval., 2014, pp. 3289-3293.

[10] N.Sager, Natural Language Information Processing .Reading ,MA,USA: AddisonWesley, 1981.

[11] R.Duggal, S.Shukla, S.Chandra, B.Shukla and S.K.Khatri "Predictive risk modelling for early hospital readmission of patients with diabetes in India," Int. J. Diabetes Develop. Countries, vol. 36, no. 4, pp. 519-528, 2016. 